\title{
Control Systems Described by a Class of Fractional Semilinear Evolution Equations and Their Relaxation Property
}

\author{
Xiaoyou Liu ${ }^{1}$ and Xi Fu \\ ${ }^{1}$ School of Mathematical Science and Computing Technology, Central South University, Changsha, \\ Hunan 410075, China \\ ${ }^{2}$ Department of Mathematics, Shaoxing University, Shaoxing, Zhejiang 312000, China
}

Correspondence should be addressed to Xiaoyou Liu, liuxiaoyou2002@hotmail.com

Received 29 May 2012; Accepted 16 December 2012

Academic Editor: Irena Lasiecka

Copyright (C) 2012 X. Liu and X. Fu. This is an open access article distributed under the Creative Commons Attribution License, which permits unrestricted use, distribution, and reproduction in any medium, provided the original work is properly cited.

\begin{abstract}
We consider a control system described by a class of fractional semilinear evolution equations in a separable reflexive Banach space. The constraint on the control is a multivalued map with nonconvex values which is lower semicontinuous with respect to the state variable. Along with the original system we also consider the system in which the constraint on the control is the upper semicontinuous convex-valued regularization of the original constraint. We obtain the existence results for the control systems and the relaxation property between the solution sets of these systems.
\end{abstract}

\section{Introduction}

Let $J=[0, b]$ and $0<\alpha<1$. We consider the following control system described by a class of fractional semilinear evolution equations of the form:

$$
\begin{gathered}
{ }^{C} D_{t}^{\alpha} x(t)=A x(t)+h(t, x(t))+g(t, x(t)) u(t), \quad t \in J, \\
x(0)=x_{0},
\end{gathered}
$$

with the mixed nonconvex constraint on the control

$$
u(t) \in U(t, x(t)) \text { a.e. on } J .
$$


Here ${ }^{C} D_{t}^{\alpha}$ is the Caputo fractional derivative of order $\alpha, b>0$ is a finite real number, $A$ is the infinitesimal generator of a strongly continuous semigroup $\{T(t), t \geq 0\}$ in a separable reflexive Banach space $X, g: J \times X \rightarrow \mathcal{L}(Y, X)(\mathcal{L}(Y, X)$ is the space of continuous linear operators from $Y$ into $X), h: J \times X \rightarrow X$ is a nonlinear function, and $U: J \times X \rightarrow 2^{Y} \backslash\{\emptyset\}$ is a multivalued mapping with closed values that is not necessarily convex. The space $Y$ is a separable, reflexive Banach space modeling the control space.

We denote by $C(J, X)$ the space of all continuous functions from $J$ into $X$ with the supremum norm given by $\|x\|_{C}=\sup _{t \in J}\|x(t)\|_{X}$ for $x \in C(J, X)$. Let $B_{X} \subseteq X$ be the open unit ball centered at zero. Consider the multivalued map

$$
\begin{gathered}
U_{\delta}(t, x)=\overline{c o}\left\{\bigcup U(t, z): z \in x+\delta B_{X}\right\}, \quad \delta>0, \\
V(t, x)=\bigcap_{\delta \downarrow 0} U_{\delta}(t, x),
\end{gathered}
$$

here $\overline{\mathrm{co}}$ stands for the closed convex hull of a set. The map (1.4) is usually called the convex upper semicontinuous regularization of $U(t, x)$.

Along with the constraint (1.2) on the control we also consider the constraint

$$
u(t) \in V(t, x(t)) \text { a.e. on } J
$$

on the control. Note that usually we have $\overline{\mathrm{co}} U(t, x) \subseteq V(t, x)$.

Definition 1.1. A solution of the control system (1.1), (1.2) is defined to be a pair $(x(\cdot), u(\cdot))$ consisting of a trajectory $x \in C(J, X)$ and a control $u \in L^{1}(J, Y)$ satisfying (1.1) and the inclusion (1.2) a.e.

A solution of the control system (1.1), (1.5) is defined similarly. We denote by $\mathcal{R}_{U}, \tau_{r_{U}}$ $\left(\mathcal{R}_{V}, \tau_{r_{V}}\right)$ the sets of all solutions, all admissible trajectories of the control system (1.1) and (1.2) (the control system (1.1) and (1.5)).

Relaxation property [1] has important ramifications in control theory. There are many papers dealing with the verification of the relaxation property for various classes of control systems. For example, we refer to [2-5] for nonlinear evolution inclusions or equations, $[6,7]$ for control problems of subdifferential type and the references therein. In this paper, we investigate this property for control systems described by fractional semilinear evolution equations. We will prove that $\tau_{r_{V}}$ is a compact set in $C(J, X)$ and

$$
\tau r_{V}=\overline{\tau r_{U}}
$$

where the bar stands for the closure in $C(J, X)$.

Fractional calculus has recently gained much attentions due to its numerous applications in science and engineering. Examples can be found in various disciplines such as mechanics, electrophysics, signal and image processing, thermodynamics, biophysics, aerodynamics, and economics, (see [8-12] for instance). For some recent results on fractional differential equations, we can refer to [13-17]. As for the study of fractional semilinear differential equations, we can refer to Zhou and Jiao [18, 19], Wang and Zhou [20] for the existence results. The issue of approximate controllability was considered by Kumar and 
Sukavanam [21], Sakthivel et al. [22]. Wang and Zhou in [23] were concerned with the optimal control settings.

\section{Preliminaries and Assumptions}

Let $J=[0, b]$ be a closed interval of the real line with the Lebesgue measure $\mu$ and the $\sigma$ algebra $\Sigma$ of $\mu$ measurable sets. The norm of the space $X$ (or $Y$ ) will be denoted by $\|\cdot\|_{X}$ (or $\left.\|\cdot\|_{Y}\right)$. For any Banach space $V$ the symbol $\omega-V$ stands for $V$ equipped with the weak $\sigma\left(V, V^{*}\right)$ topology. The same notation will be used for subsets of $V$. In all other cases we assume that $V$ and its subsets are equipped with the strong (normed) topology.

We first recall the following known definitions from fractional differential theory. For more details, please see $[11,12]$.

Definition 2.1. The fractional integral of order $\alpha$ with the lower limit zero for a function $f$ is defined as

$$
I^{\alpha} f(t)=\frac{1}{\Gamma(\alpha)} \int_{0}^{t} \frac{f(s)}{(t-s)^{1-\alpha}} d s, \quad t>0, \alpha>0
$$

provided the right hand side is point-wise defined on $[0, \infty)$, where $\Gamma(\cdot)$ is the gamma function.

Definition 2.2. The Riemann-Liouville derivative of order $\alpha$ with the lower limit zero for a function $f$ is defined as

$$
{ }^{L} D^{\alpha} f(t)=\frac{1}{\Gamma(n-\alpha)} \frac{d^{n}}{d t^{n}} \int_{0}^{t} \frac{f(s)}{(t-s)^{\alpha+1-n}} d s, \quad t>0, n-1<\alpha<n .
$$

Definition 2.3. The Caputo derivative of order $\alpha$ with the lower limit zero for a function $f$ is defined as

$$
{ }^{C} D^{\alpha} f(t)={ }^{L} D^{\alpha}\left(f(t)-\sum_{k=0}^{n-1} \frac{t^{k}}{k !} f^{(k)}(0)\right), \quad t>0, n-1<\alpha<n .
$$

If $f$ is an abstract function with values in $X$, then integrals which appear in Definitions 2.1 and 2.2 are taken in Bochner's sense.

We now proceed to some basic definitions and results from multivalued analysis. For more details on multivalued analysis, see the books [24, 25].

We use the following notations: $P_{f}(Y)$ is the set of all nonempty closed subsets of $Y$, $P_{f b}(Y)$ is the set of all nonempty, closed and bounded subsets of $Y$, and $P_{f c}(Y)$ is the set of all nonempty, closed, and convex subsets of $Y$.

On $P_{b f}(Y)$, we have a metric known as the "Hausdorff metric" and defined by

$$
h(A, B)=\max \left\{\sup _{a \in A} d(a, B), \sup _{b \in B} d(b, A)\right\}
$$


where $d(x, C)$ is the distance from a point $x$ to a set $C$. We say a multivalued map is $h$ continuous if it is continuous in the Hausdorff metric $h(\cdot, \cdot)$.

We say that a multivalued map $F: J \rightarrow P_{f}(Y)$ is measurable if $F^{-1}(E)=\{t \in J$ : $F(t) \cap E \neq \emptyset\} \in \Sigma$ for every closed set $E \subseteq Y$. If $F: J \times X \rightarrow P_{f}(Y)$, then measurability of $F$ means that $F^{-1}(E) \in \Sigma \otimes \boldsymbol{B}_{X}$, where $\Sigma \otimes \boldsymbol{B}_{X}$ is the $\sigma$-algebra of subsets in $J \times X$ generated by the sets $A \times B, A \in \Sigma, B \in \boldsymbol{B}_{X}$, and $\boldsymbol{B}_{X}$ is the $\sigma$-algebra of the Borel sets in $X$.

Suppose $V, Z$ are two Hausdorff topological spaces and $F: V \rightarrow 2^{Z} \backslash\{\emptyset\}$. We say that $F$ is lower semicontinuous in the sense of Vietoris (l.s.c. for short) at a point $x_{0} \in V$ if for any open set $W \subseteq Z, F\left(x_{0}\right) \cap W \neq \emptyset$, there is a neighborhood $O\left(x_{0}\right)$ of $x_{0}$ such that $F(x) \cap W \neq \emptyset$ for all $x \in O\left(x_{0}\right) . F$ is said to be upper semicontinuous in the sense of Vietoris (u.s.c. for short) at a point $x_{0} \in V$ if for any open set $W \subseteq Z, F\left(x_{0}\right) \subseteq W$, there is a neighborhood $O\left(x_{0}\right)$ of $x_{0}$ such that $F(x) \subseteq W$ for all $x \in O\left(x_{0}\right)$. For the properties of 1.s.c and u.s.c, see the book [24].

Besides the standard norm on $L^{q}(J, Y)$ (here $Y$ is a separable, reflexive Banach space), $1<q<\infty$, we also consider the so called weak norm:

$$
\|u(\cdot)\|_{\omega}=\sup _{0 \leq t_{1} \leq t_{2} \leq b}\left\|\int_{t_{1}}^{t_{2}} u(s) d s\right\|_{Y}, \quad \text { for } u \in L^{q}(J, Y)
$$

The space $L^{q}(J, Y)$ furnished with this norm will be denoted by $L_{\omega}^{q}(J, Y)$. The following result establishes a relation between convergence in $\omega-L^{q}(J, Y)$ and convergence in $L_{\omega}^{q}(J, Y)$.

Lemma 2.4 (see [5]). If a sequence $\left\{u_{n}\right\}_{n \geq 1} \subseteq L^{q}(J, Y)$ is bounded and converges to $u$ in $L_{\omega}^{q}(J, Y)$, then it converges to $u$ in $\omega-L^{q}(J, Y)$.

We assume the following assumptions on the data of our problems in the whole paper.

$\mathbf{H}(\mathrm{A})$ : The operator $A$ generates a strongly continuous semigroup $T(t), t \geq 0$ in $X$, and there exists a constant $M_{A} \geq 1$ such that $\sup _{t \in[0, \infty)}\|T(t)\| \leq M_{A}$. For any $t>0, T(t)$ is compact.

Remark 2.5. Let us take $X=L^{2}[0, \pi]$ and define the operator $A$ by $A \omega=\omega^{\prime \prime}$ with the domain $D(A)=\left\{\omega \in X: \omega, \omega^{\prime}\right.$ are absolutely continuous, $\omega^{\prime \prime} \in X$, and $\left.\omega(0)=\omega(\pi)=0\right\}$. Then $A \omega=-\sum_{n=1}^{\infty} n^{2}\left\langle\omega, e_{n}\right\rangle e_{n}, \omega \in D(A)$, where $e_{n}(z)=(2 / \pi)^{1 / 2} \sin n z, 0 \leq z \leq \pi, n=1,2, \ldots$ Clearly $A$ generates a compact semigroup $\{T(t): t>0\}$ in $X$ and it is given by $T(t) \omega=$ $\sum_{n=1}^{\infty} e^{-n^{2} t}\left\langle\omega, e_{n}\right\rangle e_{n}, \omega \in X$. In such a case, it is easy to see that $\mathrm{H}(\mathrm{A})$ holds [22].

$\mathbf{H}(\mathrm{g})$ : The operator $g: J \times X \rightarrow \mathcal{L}(Y, X)$ is such that

(1) the map $t \rightarrow g(t, x) u$ is measurable for all $x \in X$ and $u \in Y$;

(2) for a.e. $t \in J$, the map $x \rightarrow g^{*}(t, x) h$ is continuous for all $h \in X^{*}$, where $g^{*}(t, x)$ is the adjoint operator to $g(t, x)$;

(3) for a.e. $t \in J$ and $x \in X$

$$
\|g(t, x)\|_{\mathcal{L}(Y, X)} \leq d, \quad \text { with } d>0 .
$$

$\mathbf{H}(\mathbf{h})$ : The function $h: J \times X \rightarrow X$ of Carathéodory type satisfies: there exists a constant $0<\beta<\alpha$ such that for a.e. $t \in J$ and all $x \in X,\|h(t, x)\|_{X} \leq a_{1}(t)+c_{1}\|x\|_{X}$, where $a_{1} \in L^{1 / \beta}\left(J, \mathbb{R}^{+}\right)$and $c_{1}>0$. 
$\mathbf{H}(\mathbf{U})$ : The multivalued map $U: J \times X \rightarrow P_{f}(Y)$ is such that:

(1) $(t, x) \rightarrow U(t, x)$ is $\Sigma \otimes B_{X}$ measurable;

(2) for a.e. $t \in J$, the map $x \rightarrow U(t, x)$ is l.s.c.;

(3) for a.e. $t \in J$ and all $x \in X,\|U(t, x)\|_{Y}=\sup \left\{\|v\|_{Y}: v \in U(t, x)\right\} \leq a_{2}(t)+$ $c_{2}\|x\|_{X}$, where $a_{2} \in L^{1 / \beta}\left(J, \mathbb{R}^{+}\right)$and $c_{2}>0$.

$\mathbf{H}(\mathbf{M})$ : For any $M>0$, there exists a function $l_{M} \in L^{\infty}\left(J, \mathbb{R}^{+}\right)$such that for a.e. $t \in J$ and for any $x_{1}, x_{2} \in X,\left\|x_{1}\right\|_{X} \leq M,\left\|x_{2}\right\|_{X} \leq M$ and $u_{1} \in U\left(t, x_{1}\right)$, there is a $u_{2} \in U\left(t, x_{2}\right)$ such that

$$
\left\|g\left(t, x_{1}\right) u_{1}+h\left(t, x_{1}\right)-g\left(t, x_{2}\right) u_{2}-h\left(t, x_{2}\right)\right\|_{X} \leq l_{M}(t)\left\|x_{1}-x_{2}\right\|_{X} .
$$

We note that the condition similar to $\mathrm{H}(\mathrm{M})$ was also assumed in $[6,7]$.

From the Definitions 2.1 and 2.2 and the results obtained in [18, 19], Definition 1.1 can be rewritten in the following form.

Definition 2.6. A function $x \in C(J, X)$ is a (mild) solution of the system (1.1), (1.2) if $x(0)=x_{0}$ and there exists $u \in L^{1}(J, Y)$ such that $u(t) \in U(t, x(t))$ a.e. on $t \in J$ and

$$
x(t)=P_{\alpha}(t) x_{0}+\int_{0}^{t}(t-s)^{\alpha-1} Q_{\alpha}(t-s)(g(s, x(s)) u(s)+h(s, x(s))) d s .
$$

A similar definition can be introduced for the system (1.1) and (1.5). Here

$$
\begin{gathered}
P_{\alpha}(t)=\int_{0}^{\infty} \xi_{\alpha}(\theta) T\left(t^{\alpha} \theta\right) d \theta, \quad Q_{\alpha}(t)=\alpha \int_{0}^{\infty} \theta \xi_{\alpha}(\theta) T\left(t^{\alpha} \theta\right) d \theta, \\
\xi_{\alpha}(\theta)=\frac{1}{\alpha} \theta^{-1-(1 / \alpha)} \varpi_{\alpha}\left(\theta^{-(1 / \alpha)}\right) \geq 0, \\
\varpi_{\alpha}(\theta)=\frac{1}{\pi} \sum_{n=1}^{\infty}(-1)^{(n-1)}(\theta)^{(-n \alpha-1)} \frac{\Gamma(n \alpha+1)}{n !} \sin (n \pi \alpha), \quad \theta \in(0, \infty),
\end{gathered}
$$

and $\xi_{\alpha}$ is a probability density function defined on $(0, \infty)[26]$, that is

$$
\xi_{\alpha}(\theta) \geq 0, \quad \theta \in(0, \infty), \quad \int_{0}^{\infty} \xi_{\alpha}(\theta) d \theta=1
$$

It is not difficult to verify that

$$
\int_{0}^{\infty} \theta \xi_{\alpha}(\theta) d \theta=\frac{1}{\Gamma(1+\alpha)}
$$


Lemma 2.7 (see $[18,19])$. Let $H(A)$ hold, then the operators $P_{\alpha}$ and $Q_{\alpha}$ have the following properties.

(1) For any fixed $t \geq 0, P_{\alpha}(t)$ and $Q_{\alpha}(t)$ are linear and bounded operators, that is, for any $x \in X$,

$$
\left\|P_{\alpha}(t) x\right\|_{X} \leq M_{A}\|x\|_{X}, \quad\left\|Q_{\alpha}(t) x\right\|_{X} \leq \frac{\alpha M_{A}}{\Gamma(1+\alpha)}\|x\|_{X}
$$

(2) $\left\{P_{\alpha}(t), t \geq 0\right\}$ and $\left\{Q_{\alpha}(t), t \geq 0\right\}$ are strongly continuous.

(3) For every $t>0, P_{\alpha}(t)$ and $Q_{\alpha}(t)$ are compact operators.

Lemma 2.8 (see [27, Theorem 3.1]). Let $x(t)$ be continuous and nonnegative on $[0, b]$. If

$$
x(t) \leq \psi(t)+M \int_{0}^{t} \frac{x(s)}{(t-s)^{r}} d s, \quad 0 \leq t \leq b,
$$

where $0 \leq \gamma<1, \psi(t)$ is a non-negative, monotonic increasing continuous function on $[0, b]$ and $M$ is a positive constant, then

$$
x(t) \leq \psi(t) E_{1-\gamma}\left(M \Gamma(1-\gamma) t^{1-\gamma}\right), \quad 0 \leq t \leq b,
$$

where $E_{1-\gamma}(z)$ is the Mittag-Leffler function defined for all $\gamma<1$ by

$$
E_{1-\gamma}(z)=\sum_{n=0}^{\infty} \frac{z^{n}}{\Gamma(n(1-\gamma)+1)} .
$$

\section{Auxiliary Results}

In this section, we will give some auxiliary results needed in the proof of the main results. We begin with the a priori estimation of the trajectory of the control systems.

Lemma 3.1. For any admissible trajectory $x$ of the control system (1.1) and (1.5), that is, $x \in \tau_{r_{V}}$, there is a constant L such that

$$
\|x\|_{C} \leq L
$$

Proof. Let any $x \in \tau_{r_{V}}$, from Definition 2.6, we know that there exists a $u$ with $u(t) \in$ $V(t, x(t))$ a.e. and

$$
x(t)=P_{\alpha}(t) x_{0}+\int_{0}^{t}(t-s)^{\alpha-1} Q_{\alpha}(t-s)(g(s, x(s)) u(s)+h(s, x(s))) d s
$$


Then by Lemma 2.7, we get

$$
\begin{aligned}
\|x(t)\|_{X} \leq & M_{A}\left\|x_{0}\right\|_{X}+\frac{\alpha M_{A}}{\Gamma(1+\alpha)} \int_{0}^{t}(t-s)^{\alpha-1}\|h(s, x(s))\|_{X} d s \\
& +\frac{\alpha M_{A}}{\Gamma(1+\alpha)} \int_{0}^{t}(t-s)^{\alpha-1}\|g(s, x(s)) u(s)\|_{X} d s .
\end{aligned}
$$

From $\mathrm{H}(\mathrm{h})$ and Hölder inequality, we have

$$
\begin{aligned}
\int_{0}^{t}(t-s)^{\alpha-1}\|h(s, x(s))\|_{X} d s \leq & \int_{0}^{t}(t-s)^{\alpha-1}\left(a_{1}(s)+c_{1}\|x(s)\|_{X}\right) d s \\
\leq & {\left[\frac{(1-\beta)}{(\alpha-\beta)} b^{(\alpha-\beta) /(1-\beta)}\right]^{1-\beta}\left\|a_{1}\right\|_{L^{1 / \beta}(J)} } \\
& +c_{1} \int_{0}^{t}(t-s)^{\alpha-1}\|x(s)\|_{X} d s .
\end{aligned}
$$

Similarly, by $\mathrm{H}(\mathrm{g})(3)$ and $\mathrm{H}(\mathrm{U})(3)$,

$$
\begin{aligned}
\int_{0}^{t}(t-s)^{\alpha-1}\|g(s, x(s)) u(s)\|_{X} d s \leq & d \int_{0}^{t}(t-s)^{\alpha-1}\left(a_{2}(s)+c_{2}\|x(s)\|_{X}\right) d s \\
\leq & d\left[\frac{(1-\beta)}{(\alpha-\beta)} b^{(\alpha-\beta) /(1-\beta)}\right]^{1-\beta}\left\|a_{2}\right\|_{L^{1 / \beta}(J)} \\
& +d c_{2} \int_{0}^{t}(t-s)^{\alpha-1}\|x(s)\|_{X} d s .
\end{aligned}
$$

Combining (3.4), (3.5) with (3.3), we obtain

$$
\begin{aligned}
\|x(t)\|_{X} \leq & M_{A}\left\|x_{0}\right\|_{X}+\frac{\alpha M_{A}}{\Gamma(1+\alpha)}\left(d c_{2}+c_{1}\right) \int_{0}^{t}(t-s)^{\alpha-1}\|x(s)\|_{X} d s \\
& +\frac{\alpha M_{A}}{\Gamma(1+\alpha)}\left[\frac{(1-\beta)}{(\alpha-\beta)} b^{(\alpha-\beta) /(1-\beta)}\right]^{1-\beta}\left(\left\|a_{1}\right\|_{L^{1 / \beta}(J)}+d\left\|a_{2}\right\|_{L^{1 / \beta}(J)}\right) .
\end{aligned}
$$

From the above inequality, using the well-known singular version of the Gronwall inequality (see Lemma 2.8), we can deduce that there exists a constant $L>0$ such that $\|x\|_{C} \leq L$. Let $Q=\left\{h \in X:\|h\|_{X} \leq L\right\}$. Let $\operatorname{pr}_{L}: X \rightarrow X$ be the $L$-radial retraction, that is,

$$
\operatorname{pr}_{L}(x)= \begin{cases}x, & \|x\|_{X} \leq L \\ \frac{L x}{\|x\|_{X}}, & \|x\|_{X}>L\end{cases}
$$


This map is Lipschitz continuous. We define $U_{1}(t, x)=U\left(t, \operatorname{pr}_{L} x\right)$. Obviously, $U_{1}$ satisfies $\mathrm{H}(\mathrm{U})(1)$ and $\mathrm{H}(\mathrm{U})(2)$. Moreover, by the properties of $\mathrm{pr}_{L}$, we have for a.e. $t \in J$, all $x \in X$ and all $u \in U_{1}(t, x)$ the estimates

$$
\|u\|_{Y} \leq a_{2}(t)+c_{2} L, \quad\|u\|_{Y} \leq a_{2}(t)+c_{2}\|x\|_{X}
$$

Hence, Lemma 3.1 is still valid with $U(t, x)$ substituted by $U_{1}(t, x)$. Therefore, we assume without any loss of generality that for a.e. $t \in J$, and all $x \in X$

$$
\sup \left\{\|v\|_{Y}: v \in U(t, x)\right\} \leq \varphi(t)=a_{2}(t)+c_{2} L, \quad \text { with } \varphi \in L^{1 / \beta}\left(J, \mathbb{R}^{+}\right)
$$

Similarly, we can assume that for a.e. $t \in J$ and all $x \in X$

$$
\|h(t, x)\|_{X} \leq \gamma(t)=a_{1}(t)+c_{1} L, \quad \text { with } \gamma \in L^{1 / \beta}\left(J, \mathbb{R}^{+}\right)
$$

Let

$$
\begin{gathered}
\Upsilon_{\varphi}=\left\{u \in L^{1 / \beta}(J, Y):\|u(t)\|_{Y} \leq \varphi(t) \text { a.e. } t \in J\right\}, \\
X_{\varphi}=\left\{f \in L^{1 / \beta}(J, X):\|f(t)\|_{X} \leq d \varphi(t)+\gamma(t) \text { a.e. } t \in J\right\} .
\end{gathered}
$$

It follows from assumption $\mathrm{H}(\mathrm{g})$ that for any $h \in X^{*}$, the function $\langle h, g(t, x) u\rangle=\left\langle g^{*}(t, x) h, u\right\rangle$ is measurable in $t$ and continuous in $(x, u)$ almost everywhere. Hence, for any measurable functions $x: J \rightarrow X$ and $u: J \rightarrow Y$, the function $t \rightarrow g(t, x(t)) u(t)$ is scalarly measurable [28]. The separability of the space $X$ implies that the function $t \rightarrow g(t, x(t)) u(t)$ is measurable. Therefore, according to $\mathrm{H}(\mathrm{g})$ and $\mathrm{H}(\mathrm{h})$, for any $x \in L^{1 / \beta}(J, X)$ and $u \in L^{1 / \beta}(J, Y)$, the function $t \rightarrow g(t, x(t)) u(t)+h(t, x(t))$ is an element of the space $L^{1 / \beta}(J, X)$. Hence we can consider the operator $A: L^{1 / \beta}(J, X) \times L^{1 / \beta}(J, Y) \rightarrow L^{1 / \beta}(J, X)$ defined by the rule

$$
\mathcal{A}(x, u)(t)=g(t, x(t)) u(t)+h(t, x(t)) \text {. }
$$

Lemma 3.2. The operator $(x, u) \rightarrow \mathcal{A}(x, u)$ is sequentially continuous as an operator from $L^{1 / \beta}(J, X) \times \omega-L^{1 / \beta}(J, Y)$ into $\omega-L^{1 / \beta}(J, X)$.

Proof. Suppose that $x_{n} \rightarrow x$ in $L^{1 / \beta}(J, X)$ and $u_{n} \rightarrow u$ in $\omega-L^{1 / \beta}(J, Y)$. Take an arbitrary $u \in Y$ and any $h \in L^{1 /(1-\beta)}\left(J, X^{*}\right) . H(g)$ and the equality

$$
\left\langle h(t), g\left(t, x_{n}(t)\right) u\right\rangle=\left\langle g^{*}\left(t, x_{n}(t)\right) h(t), u\right\rangle
$$


imply that $t \rightarrow g^{*}\left(t, x_{n}(t)\right) h(t)$ is a scalarly measurable function from $J$ to $Y^{*}$. Hence it is measurable. Consider a subsequence $x_{n_{k}}, k \geq 1$, of the sequence $x_{n}, n \geq 1$, converging to $x$ a.e. in $t \in J$. By $\mathrm{H}(\mathrm{g}), \mathrm{H}(\mathrm{h})$, and (3.10), we have

$$
\begin{gathered}
g^{*}\left(t, x_{n_{k}}(t)\right) h(t) \longrightarrow g^{*}(t, x(t)) h(t) \quad \text { a.e. } t \in J \text { in } Y^{*}, \\
\left\|g^{*}\left(t, x_{n_{k}}(t)\right) h(t)\right\|_{Y^{*}} \leq d\|h(t)\|_{X^{*}} \quad \text { a.e. } t \in J, \\
h\left(t, x_{n_{k}}(t)\right) \longrightarrow h(t, x(t)) \quad \text { a.e. } t \in J \text { in } X, \\
\left\|h\left(t, x_{n_{k}}(t)\right)\right\|_{X} \leq \gamma(t) .
\end{gathered}
$$

Using the preceding four formulae and Lebesgue's theorem of dominated convergence, we obtain

$$
\begin{gathered}
g^{*}\left(t, x_{n_{k}}(t)\right) h(t) \longrightarrow g^{*}(t, x(t)) h(t) \quad \text { in } L^{1 /(1-\beta)}\left(J, Y^{*}\right), \\
h\left(t, x_{n_{k}}(t)\right) \longrightarrow h(t, x(t)) \quad \text { in } L^{1 / \beta}(J, X) .
\end{gathered}
$$

Then it follows from (3.16) that

$$
\int_{J}\left\langle g^{*}\left(t, x_{n_{k}}(t)\right) h(t), u_{n_{k}}(t)\right\rangle d t \longrightarrow \int_{J}\left\langle g^{*}(t, x(t)) h(t), u(t)\right\rangle d t
$$

Since $\langle h(t), g(t, x(t)) u(t)\rangle=\left\langle g^{*}(t, x(t)) h(t), u(t)\right\rangle$ and $h \in L^{1 /(1-\beta)}\left(J, X^{*}\right)$ is arbitrary, by (3.17) and (3.18), we deduce that

$$
\mathcal{A}\left(x_{n_{k}}, u_{n_{k}}\right) \longrightarrow \mathcal{A}(x, u) \quad \text { in } \omega-L^{1 / \beta}(J, X)
$$

It follows from (3.9), (3.10), and (3.12) that $\left\{\mathcal{A}\left(x_{n}, u_{n}\right)\right\}_{n \geq 1}$ is a subset of $X_{\varphi}$ which is a metrizable compact set in $\omega-L^{1 / \beta}(J, X)$. If the sequence $\mathcal{A}\left(x_{n}, u_{n}\right), n \geq 1$, does not converge to $\mathcal{A}(x, u)$ in $\omega-L^{1 / \beta}(J, X)$, then it has a subsequence $\mathcal{A}\left(x_{n_{i}}, u_{n_{i}}\right), i \geq 1$, such that none of its subsequences converges to $\mathcal{A}(x, u)$ in $\omega-L^{1 / \beta}(J, X)$. Applying the above arguments to this very subsequence $\left(x_{n_{i}}, u_{n_{i}}\right), i \geq 1$, we obtain a contradiction. The lemma is proved.

Lemma 3.3. For a.e. $t \in J$, the multivalued map $x \rightarrow V(t, x)$ defined by (1.4) from $X$ to $2^{\gamma}$ is u.s.c. with convex closed values.

Proof. From the definition of $V(t, x)$, it is clear that $V(t, x)$ is closed convex valued. Since $\delta \rightarrow U_{\delta}(t, x)$ is increasing (in the sense of inclusion), and letting

$$
U_{1 / n}(t, x)=\overline{\mathrm{co}}\left\{\bigcup U(t, z): z \in x+\frac{1}{n} B_{X}\right\}
$$

we obtain

$$
V(t, x)=\bigcap_{n \geq 1} U_{1 / n}(t, x)
$$


Let $x_{0} \in X$ and $W$ be an open set in $Y$ such that $V\left(t, x_{0}\right) \subseteq W$. By (3.21), we can find an $n_{0} \geq 1$ such that

$$
U_{1 / n_{0}}\left(t, x_{0}\right) \subseteq W
$$

For an arbitrary $y \in x_{0}+\left(1 / n_{0}\right) B_{X}$, we can find a $\delta>0$ such that $y+\delta B_{X} \subseteq x_{0}+\left(1 / n_{0}\right) B_{X}$. Therefore we obtain

$$
U_{\delta}(t, y) \subseteq U_{1 / n_{0}}\left(t, x_{0}\right) \subseteq W
$$

Then it is clear that $V(t, y) \subseteq W$, for all $y \in x_{0}+\left(1 / n_{0}\right) B_{X}$. This means that $x \rightarrow V(t, x)$ is u.s.c.

Let $C_{X}=\left\{z_{k}\right\}_{k \geq 1}$ be a dense countable subset of the ball $B_{X}$. We put

$$
U_{1 / n}^{k}(t, x)=U\left(t, x+\frac{1}{n} z_{k}\right), \quad z_{k} \in C_{X}
$$

Lemma 3.4. For a.e. $t \in J$, let $U_{1 / n}(t, x)$ be defined by (3.20), then we have

$$
U_{1 / n}(t, x)=\overline{\operatorname{co}}\left\{\overline{\bigcup_{k=1}^{\infty} U_{1 / n}^{k}(t, x)}\right\}
$$

where the closure is taken in $Y$.

Proof. We recall that $\overline{\mathrm{co}} \bar{A}=\overline{\mathrm{co}} A$ for any subset $A \subseteq Y$. Hence it is sufficient to prove that for a.e. $t \in J$,

$$
\overline{\bigcup_{k=1}^{\infty} U_{1 / n}^{k}(t, x)}=\left\{\overline{U U(t, z): z \in x+\frac{1}{n} B_{X}}\right\} .
$$

That the left hand side of (3.26) is contained in its right hand side is obvious. Let $w \in$ $\left\{\bigcup U(t, z): z \in x+(1 / n) B_{X}\right\}$, then $w \in U\left(t, x+(1 / n) z_{*}\right)$ for some $z_{*} \in B_{X}$. Now let $z_{m} \rightarrow z_{*},\left\{z_{m}\right\}_{m \geq 1} \subseteq C_{X}$. Since a.e. $t \in J, x \rightarrow U(t, x)$ is 1.s.c. at $x+(1 / n) z_{*}$ and $x+(1 / n) z_{m} \rightarrow x+(1 / n) z_{*}$, there is a sequence $w_{m} \in U\left(t, x+(1 / n) z_{m}\right), m \geq 1$ converging to $w$ (Proposition 1.2.6 [24]). Due to $\left\{w_{m}\right\}_{m \geq 1} \subseteq \bigcup_{k=1}^{\infty} U_{1 / n}^{k}(t, x)$, we have $w \in \overline{\bigcup_{k=1}^{\infty} U_{1 / n}^{k}(t, x)}$. Since $w$ is arbitrary, we can get $\left\{\bigcup U(t, z): z \in x+(1 / n) B_{X}\right\} \subseteq \overline{\bigcup_{k=1}^{\infty} U_{1 / n}^{k}(t, x)}$. Therefore (3.26) holds. The lemma is proved.

Now we consider the following auxiliary problem:

$$
\begin{gathered}
{ }^{C} D_{t}^{\alpha} x(t)=A x(t)+f(t), \quad t \in J=[0, b], \\
x(0)=x_{0} .
\end{gathered}
$$


It is clear that for every $f \in L^{1 / \beta}(J, X)$, (3.27) has a unique (mild) solution $S(f) \in C(J, X)$ which is given by

$$
S(f)(t)=P_{\alpha}(t) x_{0}+\int_{0}^{t}(t-s)^{\alpha-1} Q_{\alpha}(t-s) f(s) d s
$$

The following lemma describes a property of the solution map $S$ which is crucial in our investigation.

Lemma 3.5. The solution map $S: X_{\varphi} \rightarrow C(J, X)$ is continuous from $\omega-X_{\varphi}$ to $C(J, X)$.

Proof. Consider the operator $H: L^{1 / \beta}(J, X) \rightarrow C(J, X)$ defined by

$$
H(f)(t)=\int_{0}^{t}(t-s)^{\alpha-1} Q_{\alpha}(t-s) f(s) d s
$$

We know $H$ is linear. From simple calculation, one has

$$
\|H(f)\|_{C} \leq \frac{\alpha M_{A}}{\Gamma(1+\alpha)}\left[\frac{(1-\beta)}{(\alpha-\beta)} b^{(\alpha-\beta) /(1-\beta)}\right]^{1-\beta}\|f\|_{L^{1 / \beta}(J, X)^{\prime}}
$$

that is, the operator $H$ is continuous from $L^{1 / \beta}(J, X)$ into $C(J, X)$, hence $H$ is also continuous from $\omega-L^{1 / \beta}(J, X)$ into $\omega-C(J, X)$.

Let any $B \in P_{b}\left(L^{1 / \beta}(J, X)\right)$ and suppose that for any $f \in B,\|f\|_{L^{1 / \beta}(J, X)} \leq K(K>0$ is a constant). Next we will show that $H$ is completely continuous.

(a) From (3.30), we know that $\|H(f)(t)\|_{X}$ is uniformly bounded for any $t \in J$ and $f \in B$.

(b) $H$ is equicontinuous on $B$. Let $0 \leq t_{1}<t_{2} \leq b$ and any $f \in B$, we get

$$
\begin{aligned}
& \left\|H(f)\left(t_{2}\right)-H(f)\left(t_{1}\right)\right\|_{X} \\
& =\left\|\int_{0}^{t_{2}}\left(t_{2}-s\right)^{\alpha-1} Q_{\alpha}\left(t_{2}-s\right) f(s) d s-\int_{0}^{t_{1}}\left(t_{1}-s\right)^{\alpha-1} Q_{\alpha}\left(t_{1}-s\right) f(s) d s\right\|_{X} \\
& \leq\left\|\int_{t_{1}}^{t_{2}}\left(t_{2}-s\right)^{\alpha-1} Q_{\alpha}\left(t_{2}-s\right) f(s) d s\right\|_{X} \cdots \text { denoted by } I_{1} \\
& \quad+\left\|\int_{0}^{t_{1}}\left(\left(t_{2}-s\right)^{\alpha-1}-\left(t_{1}-s\right)^{\alpha-1}\right) Q_{\alpha}\left(t_{2}-s\right) f(s) d s\right\|_{X} \cdots \text { denoted by } I_{2} \\
& \quad+\left\|\int_{0}^{t_{1}}\left(t_{1}-s\right)^{\alpha-1}\left(Q_{\alpha}\left(t_{2}-s\right)-Q_{\alpha}\left(t_{1}-s\right)\right) f(s) d s\right\|_{X} \cdots \text { denoted by } I_{3} .
\end{aligned}
$$


By using analogous arguments as in Lemma 3.1, we find

$$
\begin{gathered}
I_{1} \leq \frac{\alpha M_{A}}{\Gamma(1+\alpha)}\left[\frac{(1-\beta)}{(\alpha-\beta)}\right]^{1-\beta} K\left(t_{2}-t_{1}\right)^{\alpha-\beta}, \\
I_{2} \leq \frac{\alpha M_{A}}{\Gamma(1+\alpha)}\left(\int_{0}^{t_{1}}\left(\left(t_{1}-s\right)^{\alpha-1}-\left(t_{2}-s\right)^{\alpha-1}\right)^{1 /(1-\beta)} d s\right)^{1-\beta} K \\
\leq \frac{\alpha M_{A}}{\Gamma(1+\alpha)}\left(\int_{0}^{t_{1}}\left(\left(t_{1}-s\right)^{(\alpha-1) /(1-\beta)}-\left(t_{2}-s\right)^{(\alpha-1) /(1-\beta)}\right) d s\right)^{1-\beta} K \\
=\frac{\alpha M_{A}}{\Gamma(1+\alpha)}\left[\frac{(1-\beta)}{(\alpha-\beta)}\right]^{1-\beta}\left(t_{1}^{(\alpha-\beta) /(1-\beta)}-t_{2}^{(\alpha-\beta) /(1-\beta)}+\left(t_{2}-t_{1}\right)^{(\alpha-\beta) /(1-\beta)}\right)^{1-\beta} K \\
\leq \frac{2 \alpha M_{A}}{\Gamma(1+\alpha)}\left[\frac{(1-\beta)}{(\alpha-\beta)}\right]^{1-\beta}\left(t_{2}-t_{1}\right)^{\alpha-\beta} K .
\end{gathered}
$$

For $t_{1}=0,0<t_{2} \leq b$, it is easy to see that $I_{3}=0$. For $t_{1}>0$ and $\epsilon>0$ be enough small, we have

$$
\begin{aligned}
I_{3} \leq & \left\|\int_{0}^{t_{1}-\epsilon}\left(t_{1}-s\right)^{\alpha-1}\left(Q_{\alpha}\left(t_{2}-s\right)-Q_{\alpha}\left(t_{1}-s\right)\right) f(s) d s\right\|_{X} \\
& +\left\|\int_{t_{1}-\epsilon}^{t_{1}}\left(t_{1}-s\right)^{\alpha-1}\left(Q_{\alpha}\left(t_{2}-s\right)-Q_{\alpha}\left(t_{1}-s\right)\right) f(s) d s\right\|_{X} \\
\leq & \sup _{s \in\left[0, t_{1}-\epsilon\right]}\left\|Q_{\alpha}\left(t_{2}-s\right)-Q_{\alpha}\left(t_{1}-s\right)\right\|\left[\frac{(1-\beta)}{(\alpha-\beta)}\right]^{1-\beta} \\
& \times\left(t_{1}^{(\alpha-\beta) /(1-\beta)}-\epsilon^{(\alpha-\beta) /(1-\beta)}\right)^{1-\beta} K \\
& +\frac{2 \alpha M_{A}}{\Gamma(1+\alpha)}\left[\frac{(1-\beta)}{(\alpha-\beta)}\right]^{1-\beta} \epsilon^{\alpha-\beta} K .
\end{aligned}
$$

Combining the estimations for $I_{1}, I_{2}$, and $I_{3}$, and letting $t_{2} \rightarrow t_{1}$ and $\epsilon \rightarrow 0$ in $I_{3}$, we obtain that $H$ is equicontinuous. For more details, please see [19].

(c) The set $\Pi(t)=\{H(f)(t): f \in B\}$ is relatively compact in $X$. Clearly, $\Pi(0)=\{0\}$ is compact, and hence, it is only necessary to consider $t>0$. For each $h \in(0, t)$, $t \in(0, b], f \in B$ and $\delta>0$ be arbitrary, we define

$$
\Pi_{h, \delta}(t)=\left\{H_{h, \delta}(f)(t): f \in B\right\}
$$


where

$$
\begin{aligned}
H_{h, \delta}(f)(t) & =\alpha \int_{0}^{t-h} \int_{\delta}^{\infty} \theta(t-s)^{\alpha-1} \xi_{\alpha}(\theta) T\left((t-s)^{\alpha} \theta\right) f(s) d \theta d s \\
& =\alpha \int_{0}^{t-h} \int_{\delta}^{\infty} \theta(t-s)^{\alpha-1} \xi_{\alpha}(\theta) T\left(h^{\alpha} \delta\right) T\left((t-s)^{\alpha} \theta-h^{\alpha} \delta\right) f(s) d \theta d s \\
& =\alpha T\left(h^{\alpha} \delta\right) \int_{0}^{t-h} \int_{\delta}^{\infty} \theta(t-s)^{\alpha-1} \xi_{\alpha}(\theta) T\left((t-s)^{\alpha} \theta-h^{\alpha} \delta\right) f(s) d \theta d s .
\end{aligned}
$$

From the compactness of $T\left(h^{\alpha} \delta\right)\left(h^{\alpha} \delta>0\right)$, we obtain that the set $\Pi_{h, \delta}(t)$ is relatively compact in $X$ for any $h \in(0, t)$ and $\delta>0$. Moreover, we have

$$
\begin{aligned}
& \left\|H(f)(t)-H_{h, \delta}(f)(t)\right\|_{X} \\
& =\alpha \| \int_{0}^{t} \int_{0}^{\delta} \theta(t-s)^{\alpha-1} \xi_{\alpha}(\theta) T\left((t-s)^{\alpha} \theta\right) f(s) d \theta d s \\
& \quad+\int_{0}^{t} \int_{\delta}^{\infty} \theta(t-s)^{\alpha-1} \xi_{\alpha}(\theta) T\left((t-s)^{\alpha} \theta\right) f(s) d \theta d s \\
& \quad-\int_{0}^{t-h} \int_{\delta}^{\infty} \theta(t-s)^{\alpha-1} \xi_{\alpha}(\theta) T\left((t-s)^{\alpha} \theta\right) f(s) d \theta d s \|_{X} \\
& \leq \alpha\left\|\int_{0}^{t} \int_{0}^{\delta} \theta(t-s)^{\alpha-1} \xi_{\alpha}(\theta) T\left((t-s)^{\alpha} \theta\right) f(s) d \theta d s\right\|_{X} \\
& \quad+\alpha \int_{t-h}^{t} \int_{\delta}^{\infty} \theta(t-s)^{\alpha-1} \xi_{\alpha}(\theta) T\left((t-s)^{\alpha} \theta\right) f(s) d \theta d s \|_{X} \\
& \leq M_{A} \alpha\left(\int_{0}^{t}(t-s)^{(\alpha-1) /(1-\beta)} d s\right)^{1-\beta}\|f\|_{L^{1 / \beta}(J, X)} \int_{0}^{\delta} \theta \xi_{\alpha}(\theta) d \theta \\
& \quad+M_{A} \alpha\left(\int_{t-h}^{t}(t-s)^{(\alpha-1) /(1-\beta)} d s\right)^{1-\beta}\|f\|_{L^{1 / \beta}(J, X)} \int_{\delta}^{\infty} \theta \xi_{\alpha}(\theta) d \theta \\
& \leq M_{A} K \alpha\left[\frac{(1-\beta)}{(\alpha-\beta)}\right]^{1-\beta}\left(b^{\alpha-\beta} \int_{0}^{\delta} \theta \xi_{\alpha}(\theta) d \theta+\frac{1}{\Gamma(1+\alpha)} h^{\alpha-\beta}\right) .
\end{aligned}
$$

In virtue of (2.11), the last term of the preceding inequality tends to zero as $h \rightarrow 0$ and $\delta \rightarrow 0$. Therefore, there exist relatively compact sets arbitrarily close to the set $\Pi(t), t>0$. Hence the set $\Pi(t), t>0$ is also relatively compact in $X$.

Since $X_{\varphi}$ is a convex compact metrizable subset of $\omega-L^{1 / \beta}(J, X)$, it suffices to prove the sequential continuity of the map $S$. Now let $\left\{f_{n}\right\}_{n \geq 1} \subseteq X_{\varphi}$ such that

$$
f_{n} \longrightarrow f \quad \text { in } \omega-L^{1 / \beta}(J, X), f \in X_{\varphi}
$$


By the property of the operator $H$, we have $H\left(f_{n}\right) \rightarrow H(f)$ in $\omega-C(J, X)$. Since $\left\{f_{n}\right\}_{n \geq 1}$ is bounded, there is a subsequence $\left\{f_{n_{k}}\right\}_{k \geq 1}$ of the sequence $\left\{f_{n}\right\}_{n \geq 1}$ such that $H\left(f_{n_{k}}\right) \rightarrow z$ in $C(J, X)$ for some $z \in C(J, X)$. From the facts that

$$
H\left(f_{n}\right) \longrightarrow H(f) \quad \text { in } \omega-C(J, X), \quad H\left(f_{n_{k}}\right) \longrightarrow z \quad \text { in } C(J, X)
$$

we obtain that $z=H(f)$ and $H\left(f_{n}\right) \rightarrow H(f)$ in $C(J, X)$.

From the definitions of the operators $S$ and $H$, we have that $S(f)(t)=P_{\alpha}(t) x_{0}+$ $H(f)(t)$. Then due to the arguments above, we have $S\left(f_{n}\right) \rightarrow S(f)$ in $C(J, X)$. This completes the proof of the lemma.

\section{Existence Results for the Control Systems}

In the present section, we are interested in the existence results for the control systems (1.1), (1.2) and (1.1), (1.5).

Let $\Lambda=S\left(X_{\varphi}\right)$, from Lemma 3.5, we have that $\Lambda$ is a compact subset of $C(J, X)$. It follows from formulae (3.9), (3.10), and (3.12) that $\tau_{r_{U}} \subseteq \tau_{r_{V}} \subseteq \Lambda$. Let $\bar{U}: C(J, X) \rightarrow$ $2^{L^{1 / \beta}(J, Y)}$ be defined by

$$
\bar{U}(x)=\{h: J \longrightarrow Y \text { measurable }: h(t) \in U(t, x(t)) \text { a.e. }\}, \quad x \in C(J, X) .
$$

Theorem 4.1. The set $\mathcal{R}_{U}$ is nonempty and the set $\mathcal{R}_{V}$ is a compact subset of the space $C(J, X) \times \omega-$ $L^{1 / \beta}(J, Y)$.

Proof. By the hypothesis $\mathrm{H}(\mathrm{U})(1)$, we have that for any measurable function $x: J \rightarrow X$, the map $t \rightarrow U(t, x(t))$ is measurable and has closed values. Therefore it has measurable selectors [29]. So the operator $\bar{U}$ is well defined and its values are closed decomposable subsets of $L^{1 / \beta}(J, Y)$. We claim that $x \rightarrow \bar{U}(x)$ is 1.s.c. Let $x_{*} \in C(J, X), h_{*} \in \bar{U}\left(x_{*}\right)$ and let $\left\{x_{n}\right\}_{n \geq 1} \subseteq C(J, X)$ be a sequence converging to $x_{*}$. It follows from Lemma 3.2 in [30] that there exists a sequence $h_{n} \in \bar{U}\left(x_{n}\right)$ such that

$$
\left\|h_{*}(t)-h_{n}(t)\right\|_{Y} \leq d_{Y}\left(h_{*}(t), U\left(t, x_{n}(t)\right)\right)+\frac{1}{n}, \quad \text { a.e. } t \in J .
$$

Since the map $y \rightarrow U(t, y)$ is 1.s.c., by the Proposition 1.2.26 in [24], the function $y \rightarrow$ $d_{Y}\left(h_{*}(t), U(t, y)\right)$ is u.s.c. for a.e. $t \in J$. It follows from (4.2) that for a.e. $t \in J$

$$
\begin{aligned}
\lim _{n \rightarrow \infty}\left\|h_{*}(t)-h_{n}(t)\right\|_{Y} & \leq \varlimsup_{n \rightarrow \infty} d_{Y}\left(h_{*}(t), U\left(t, x_{n}(t)\right)\right) \\
& \leq d_{Y}\left(h_{*}(t), U\left(t, x_{*}(t)\right)\right)=0 .
\end{aligned}
$$

This together with (3.9) implies that $h_{n} \rightarrow h_{*}$ in $L^{1 / \beta}(J, Y)$. Therefore the map $x \rightarrow \bar{U}(x)$ is 1.s.c. By Proposition 2.2 in [31], there is a continuous function $m: \Lambda \rightarrow L^{1 / \beta}(J, Y)$ such that

$$
m(x) \in \bar{U}(x), \quad \forall x \in \Lambda
$$


Consider the map $D: L^{1 / \beta}(J, X) \rightarrow L^{1 / \beta}(J, Y)$ defined by $p(f)=m(S(f))$. Due to Lemma 3.5 and the continuity of $m$, the map $D$ is continuous from $\omega-X_{\varphi}$ into $L^{1 / \beta}(J, Y)$. Then by Lemma 3.2, we deduce that the map $f \rightarrow \mathcal{A}(S(f), D(f))$ is continuous from $\omega-X_{\varphi}$ into $\omega-L^{1 / \beta}(J, X)$. It follows from (3.9), (3.10), (3.12), and (3.13) that $\mathcal{A}(S(f), p(f)) \in X_{\varphi}$ for every $f \in X_{\varphi}$. Therefore, the map $f \rightarrow \mathcal{A}(S(f), D(f))$ is continuous from $\omega-X_{\varphi}$ into $\omega-X_{\varphi}$. Since $\omega-X_{\varphi}$ is a convex metrizable compact set in $\omega-L^{1 / \beta}(J, X)$, Schauder's fixed point theorem implies that this map has a fixed point $f_{*} \in X_{\varphi}$, that is, $f_{*}=\mathscr{A}\left(S\left(f_{*}\right), D\left(f_{*}\right)\right)$. Let $u_{*}=P\left(f_{*}\right)$ and $x_{*}=S\left(f_{*}\right)$, then we have $u_{*}=m\left(x_{*}\right)$ and $f_{*}=\mathcal{A}\left(x_{*}, u_{*}\right)$. That means

$$
\begin{gathered}
x_{*}(t)=P_{\alpha}(t) x_{0}+\int_{0}^{t}(t-s)^{\alpha-1} Q_{\alpha}(t-s)\left(g\left(s, x_{*}(s)\right) u_{*}(s)+h\left(s, x_{*}(s)\right)\right) d s, \\
u_{*}(t) \in U\left(t, x_{*}(t)\right), \quad \text { a.e. } t \in J,
\end{gathered}
$$

which implies that $\left(x_{*}(\cdot), u_{*}(\cdot)\right)$ is a solution of the control system (1.1) and (1.2). Hence $\mathcal{R}_{U}$ is nonempty.

It is easy to see that $R_{V} \subseteq \Lambda \times Y_{\varphi}$. Since $\Lambda$ is compact in $C(J, X)$ and $Y_{\varphi}$ is metrizable convex compact in $\omega-L^{1 / \beta}(J, Y)$, we have that $R_{V}$ is relatively compact in $C(J, X) \times \omega-$ $L^{1 / \beta}(J, Y)$. Hence to complete the proof of this theorem, it is sufficient to prove that $\mathcal{R}_{V}$ is sequentially closed in $C(J, X) \times \omega-L^{1 / \beta}(J, Y)$.

Let $\left\{\left(x_{n}(\cdot), u_{n}(\cdot)\right)\right\}_{n \geq 1} \subseteq \mathcal{R}_{V}$ be a sequence converging to $(x(\cdot), u(\cdot))$ in $C(J, X) \times \omega-$ $L^{1 / \beta}(J, Y)$. Denote

$$
\begin{gathered}
f_{n}(t)=g\left(t, x_{n}(t)\right) u_{n}(t)+h\left(t, x_{n}(t)\right), \\
f(t)=g(t, x(t)) u(t)+h(t, x(t)) .
\end{gathered}
$$

According to Lemma 3.2, $f_{n} \rightarrow f$ in $\omega-L^{1 / \beta}(J, X)$. Since $f_{n} \in X_{\varphi}$ and $x_{n}=S\left(f_{n}\right), n \geq 1$, Lemma 3.5 implies that

$$
x=S(f) .
$$

Hence, to prove that $(x(\cdot), u(\cdot)) \in \mathcal{R}_{V}$, we only need to verify that $u(t) \in V(t, x(t))$ a.e. $t \in J$.

Since $u_{n} \rightarrow u$ in $\omega-L^{1 / \beta}(J, Y)$, by Mazur's theorem we have

$$
u(t) \in \bigcap_{n=1}^{\infty} \overline{\mathrm{co}}\left(\bigcup_{k=n}^{\infty} u_{k}(t)\right), \quad \text { for a.e. } t \in J
$$

From Lemma 3.3, we have that for a.e. $t \in J$, the map $x \rightarrow V(t, x) \in P_{f c}(Y)$ is u.s.c., then by Proposition 1.2.61 in [24], the map $x \rightarrow V(t, x) \in P_{f c}(Y)$ is $h$-upper semicontinuous. Therefore from assertion (b) of Proposition 1.2.86 in [24], the map $x \rightarrow V(t, x)$ has property $Q$. Hence we have

$$
\bigcap_{n=1}^{\infty} \overline{\mathrm{co}}\left(\bigcup_{k=n}^{\infty} V\left(t, x_{k}(t)\right)\right) \subseteq V(t, x(t)), \quad \text { for a.e. } t \in J .
$$


In virtue of (4.8) and (4.9), and for a.e. $t \in J, u_{n}(t) \in V\left(t, x_{n}(t)\right), n \geq 1$, we obtain that $u(t) \in V(t, x(t))$ a.e. $t \in J$. This means that $R_{V}$ is compact in $C(J, X) \times \omega-L^{1 / \beta}(J, Y)$. The proof is complete.

\section{Main Results}

In this section, we will prove the relaxation result. But first, we give a lemma which is important in the proof of our relaxation theorem.

Lemma 5.1. For any pair $\left(x_{*}(\cdot), u_{*}(\cdot)\right) \in \mathcal{R}_{V}$, there exists a sequence of simple functions $y_{n}: J \rightarrow X$ and a sequence $v_{n} \in L^{1 / \beta}(J, Y), n \geq 1$, such that

$$
\begin{gathered}
\left\|y_{n}(t)\right\|_{X} \leq \frac{1}{n}, \quad t \in J, n \geq 1, \\
v_{n}(t) \in U\left(t, x_{*}(t)+y_{n}(t)\right), \quad t \in J, \\
v_{n} \longrightarrow u_{*} \text { in } \omega-L^{1 / \beta}(J, Y) .
\end{gathered}
$$

Proof. Let $\left(x_{*}(\cdot), u_{*}(\cdot)\right) \in \mathcal{R}_{V}$. From Lemma 3.4, we have that for a.e. $t \in J, n \geq 1$

$$
u_{*}(t) \in V\left(t, x_{*}(t)\right) \subseteq U_{1 / n}\left(t, x_{*}(t)\right)=\overline{\mathrm{co}}\left\{\overline{\bigcup_{k=1}^{\infty} U_{1 / n}^{k}\left(t, x_{*}(t)\right)}\right\} .
$$

The map $t \rightarrow \overline{\bigcup_{k=1}^{\infty} U_{1 / n}^{k}\left(t, x_{*}(t)\right)}$ is measurable (see Propositions 2.3 and 2.6 in [29]) and, by (3.9), is integrally bounded. Therefore, from (5.4) and Theorem 2.2 in [32], we have that there exists an $f_{n} \in L^{1 / \beta}(J, Y)$ such that

$$
f_{n}(t) \in \overline{\bigcup_{k=1}^{\infty} U_{1 / n}^{k}\left(t, x_{*}(t)\right)} \quad \text { a.e. } t \in J, \quad\left\|u_{*}-f_{n}\right\|_{\omega} \leq \frac{1}{n} .
$$

We know that the map $t \rightarrow U_{1 / n}^{k}\left(t, x_{*}(t)\right)$ is measurable and its value are closed, then following Theorem 5.6 in [29] (also Proposition 2.2.3 in [24]), there exists a sequence of measurable selectors $f_{k}^{m}(t) \in U_{1 / n}^{k}\left(t, x_{*}(t)\right), t \in J, m \geq 1$ such that

$$
U_{1 / n}^{k}\left(t, x_{*}(t)\right)=U\left(t, x_{*}(t)+\frac{1}{n} z_{k}\right)=\overline{\bigcup_{m=1}^{\infty} f_{k}^{m}(t)}, \quad \text { for } t \in J, k \geq 1
$$

Therefore we have

$$
\overline{\bigcup_{k=1}^{\infty} u_{1 / n}^{k}\left(t, x_{*}(t)\right)}=\overline{\bigcup_{k=1}^{\infty} U\left(t, x_{*}(t)+\frac{1}{n} z_{k}\right)}=\overline{\bigcup_{k=1}^{\infty} \bigcup_{m=1}^{\infty} f_{k}^{m}(t)}, \quad \text { for } t \in T \text {. }
$$


From (5.5) and (5.7), according to Lemma 1.3 in [33] (also see Proposition 2.3.6 [24]), there is a finite measurable partition $J_{1}, J_{2}, \ldots, J_{l(n)}$ of $J$ such that

$$
\left(\int_{J}\left\|f_{n}(t)-\sum_{i=1}^{l(n)} X\left(J_{i}\right) f_{k_{i}}^{m_{i}}(t)\right\|_{Y}^{1 / \beta} d t\right)^{\beta} \leq \frac{1}{n^{\prime}}
$$

where $\chi\left(J_{i}\right)$ is the characteristic function of the set $J_{i}$. Now let

$$
v_{n}(t)=\sum_{i=1}^{l(n)} \chi\left(J_{i}\right) f_{k_{i}}^{m_{i}}(t), \quad y_{n}(t)=\sum_{i=1}^{l(n)} \chi\left(J_{i}\right) \frac{1}{n} z_{k_{i}} .
$$

Formula (5.9) implies that $y_{n}$ is a simple function, $v_{n} \in L^{1 / \beta}(J, Y)$ and (5.1), (5.2) hold. By Lemma 2.4, (5.5) and (5.8), we obtain that (5.3) holds. The lemma is proved.

Now we are ready to present our main result.

Theorem 5.2. The set $\tau_{r_{V}}$ is compact in $C(J, X)$ and the following relation holds

$$
\tau r_{V}=\overline{\tau r_{U}}
$$

where the bar stands for the closure in $C(J, X)$.

Proof. Let $\left(x_{*}(\cdot), u_{*}(\cdot)\right) \in \mathcal{R}_{V}$ and $\left\{v_{n}\right\}_{n \geq 1},\left\{y_{n}\right\}_{n \geq 1}$ be given as in Lemma 5.1. Put $Q=\{h \in X$ : $\left.\|h\|_{X} \leq L\right\}$, for fixed $n \geq 1$, we consider the function defined by

$$
\begin{aligned}
r_{n}(t, x, u)=\| & g\left(t, x_{*}(t)+y_{n}(t)\right) v_{n}(t)+h\left(t, x_{*}(t)+y_{n}(t)\right) \\
& -g(t, x) u-h(t, x)\left\|_{X}-l_{L+1}(t)\right\| x_{*}(t)+y_{n}(t)-x \|_{X} .
\end{aligned}
$$

It is clear that the function $t \rightarrow r_{n}(t, x, u)$ is measurable and the function $(x, u) \rightarrow r_{n}(t, x, u)$ is continuous (in view of $\mathrm{H}(\mathrm{g})$ and the fact that if $x: J \rightarrow X$ is a measurable function, $\|x(t)\|_{X}$ is a measurable real-valued function). According to the Theorem 2.4 in [34], there exists a sequence of nested (in the sense of inclusion) closed sets $J_{k} \subseteq J, k \geq 1, \mu\left(J \backslash \cup_{k=1}^{\infty} J_{k}\right)=0$ such that the map $(t, x) \rightarrow U(t, x)$ is 1.s.c. on $J_{k} \times Q$ and $r_{n}(t, x, u)$ is continuous on $J_{k} \times Q \times Y$. Let the multivalued map $H_{n}: J \times Q \rightarrow Y$ be defined by

$$
H_{n}(t, x)=\left\{u \in Y: r_{n}(t, x, u)-\frac{1}{n}<0\right\}
$$

For every $k \geq 1$ the graph of the map $H_{n}(t, x)$ is an open subset of $J_{k} \times Q \times Y$. Let the map $U_{n}: J \times Q \rightarrow Y$ be defined by

$$
U_{n}(t, x)=H_{n}(t, x) \cap U(t, x)
$$


Hypothesis $\mathrm{H}(\mathrm{M})$ together with (5.12) implies that $U_{n}(t, x)$ is nonempty for a.e. $t \in J$ and all $x \in Q$. Since the map $U(t, x)$ is 1.s.c. on $J_{k} \times Q, k \geq 1$, and the graph of the map $H_{n}(t, x)$ is an open subset of $J_{k} \times Q \times Y, k \geq 1$, then according to Proposition 1.2.47 in [24], we obtain that the $\operatorname{map} U_{n}(t, x)$ is 1.s.c. on $J_{k} \times Q, k \geq 1$. Hence the map $\overline{U_{n}}(t, x)=\overline{U_{n}(t, x)}$ is 1.s.c. on $J_{k} \times Q, k \geq 1$. Therefore, for every continuous function $x: J \rightarrow Q$ the map $t \rightarrow \overline{U_{n}}(t, x(t))$ is measurable and the map $x \rightarrow \overline{U_{n}}(t, x)$ is l.s.c. on $Q$ for a.e. $t \in J$.

It is clear that $\overline{U_{n}}(t, x) \subseteq U(t, x)$. Consider the system (1.1) with the constraint $\overline{U_{n}}(t, x(t))$ on the control. The arguments used in the proof of the Theorem 4.1 enable us to obtain the existence of a solution $\left(x_{n}(\cdot), u_{n}(\cdot)\right) \in \mathcal{R}_{U}$ and

$$
\begin{gathered}
\| g\left(t, x_{*}(t)+y_{n}(t)\right) v_{n}(t)+h\left(t, x_{*}(t)+y_{n}(t)\right)-g\left(t, x_{n}(t)\right) u_{n}(t) \\
-h\left(t, x_{n}(t)\right)\left\|_{X}-l_{L+1}(t)\right\| x_{*}(t)+y_{n}(t)-x_{n}(t) \|_{X}-\frac{1}{n} \leq 0 .
\end{gathered}
$$

Now by $\left(x_{*}(\cdot), u_{*}(\cdot)\right) \in \mathcal{R}_{V}$ and $\left(x_{n}(\cdot), u_{n}(\cdot)\right) \in \mathcal{R}_{U}, n \geq 1$, we have

$$
\begin{aligned}
& x_{*}(t)=P_{\alpha}(t) x_{0}+\int_{0}^{t}(t-s)^{\alpha-1} Q_{\alpha}(t-s)\left(g\left(s, x_{*}(s)\right) u_{*}(s)+h\left(s, x_{*}(s)\right)\right) d s, \\
& x_{n}(t)=P_{\alpha}(t) x_{0}+\int_{0}^{t}(t-s)^{\alpha-1} Q_{\alpha}(t-s)\left(g\left(s, x_{n}(s)\right) u_{n}(s)+h\left(s, x_{n}(s)\right)\right) d s .
\end{aligned}
$$

Theorem 4.1 and $\mathcal{R}_{U} \subseteq \mathcal{R}_{V}$ imply that we can assume, possibly up to a subsequence, that the sequence $\left(x_{n}(\cdot), u_{n}(\cdot)\right) \rightarrow(\bar{x}(\cdot), \bar{u}(\cdot)) \in \mathcal{R}_{V}$ in $C(J, X) \times \omega-L^{1 / \beta}(J, Y)$. Subtracting (5.15) from (5.16), we have

$$
\begin{aligned}
\left\|x_{n}(t)-x_{*}(t)\right\|_{X}=\| & H\left(g\left(\cdot, x_{n}(\cdot)\right) u_{n}(\cdot)+h\left(\cdot, x_{n}(\cdot)\right)\right)(t) \\
& -H\left(g\left(\cdot, x_{*}(\cdot)\right) u_{*}(\cdot)-h\left(\cdot, x_{*}(\cdot)\right)\right)(t) \|_{X} \\
\leq \| & H\left(g\left(\cdot, x_{*}(\cdot)+y_{n}(\cdot)\right) v_{n}(\cdot)+h\left(\cdot, x_{*}(\cdot)+y_{n}(\cdot)\right)-g\left(\cdot, x_{*}(\cdot)\right) u_{*}(\cdot)\right. \\
- & \left.h\left(\cdot, x_{*}(\cdot)\right)\right)(t) \|_{X} \cdots \text { denoted by } D_{1} \\
+ & \| H\left(g\left(\cdot, x_{n}(\cdot)\right) u_{n}(\cdot)+h\left(\cdot, x_{n}(\cdot)\right)-g\left(\cdot, x_{*}(\cdot)+y_{n}(\cdot)\right) v_{n}(\cdot)\right. \\
& \left.-h\left(\cdot, x_{*}(\cdot)+y_{n}(\cdot)\right)\right)(t) \|_{X} \cdots \text { denoted by } D_{2} .
\end{aligned}
$$

Here the linear operator $H$ is defined by (3.29). In virtue of (5.1), we have $x_{*}+y_{n} \rightarrow x_{*}$ in $L^{1 / \beta}(J, X)$. Since $v_{n} \rightarrow u_{*}$ in $\omega-L^{1 / \beta}(J, Y)$ (see (5.3)), from Lemma 3.2 we have that $\mathcal{A}\left(x_{*}+\right.$ $\left.y_{n}, v_{n}\right) \rightarrow \mathcal{A}\left(x_{*}, u_{*}\right)$ in $\omega-L^{1 / \beta}(J, X)$. By the property of the operator $H$ in Lemma 3.5, we obtain

$$
D_{1} \longrightarrow 0 \text { for every } t \in J
$$


Due to (5.14), we have

$$
\begin{aligned}
D_{2} \leq & \frac{\alpha M_{A}}{\Gamma(1+\alpha)} \int_{0}^{t}(t-s)^{\alpha-1}\left(l_{L+1}(s)\left\|x_{*}(s)+y_{n}(s)-x_{n}(s)\right\|_{X}+\frac{1}{n}\right) d s \\
\leq & \frac{\alpha M_{A}\left\|l_{L+1}\right\|_{L^{\infty}(J)}}{\Gamma(1+\alpha)} \int_{0}^{t}(t-s)^{\alpha-1} Q_{\alpha}(t-s)\left\|x_{*}(s)+y_{n}(s)-x_{n}(s)\right\|_{X} d s \\
& +\frac{\alpha M_{A} b^{\alpha}}{n \alpha \Gamma(1+\alpha)} .
\end{aligned}
$$

Note that $\left\|x_{*}(t)\right\|_{X} \leq L,\left\|x_{n}(t)\right\|_{X} \leq L$ for any $n \geq 1, t \in J$. Combining (5.18), (5.19) with (5.17), let $n \rightarrow \infty$, we get

$$
\left\|\bar{x}(t)-x_{*}(t)\right\|_{X} \leq \frac{\alpha M_{A}\left\|l_{L+1}\right\|_{L^{\infty}(J)}}{\Gamma(1+\alpha)} \int_{0}^{t}(t-s)^{\alpha-1} Q_{\alpha}(t-s)\left\|x_{*}(s)-\bar{x}(s)\right\|_{X} d s
$$

This together with Lemma 2.8 implies that $x_{*}=\bar{x}$. Hence we have that the sequence $x_{n} \in \tau_{r_{U}}$, $n \geq 1$, converges to $x_{*} \in \tau_{r_{V}}$ in $C(J, X)$. From Theorem 4.1, we know that $\tau_{r_{V}}$ is compact in $C(J, X)$. Now it follows from $\tau_{r_{U}} \subseteq \tau_{r_{V}}$ and the proof above that the relation (5.10) holds. The proof is complete.

\section{Acknowledgment}

This project is supported by Tianyuan foundation 11226096 .

\section{References}

[1] S. Hu and N. S. Papageorgiou, Handbook of Multivalued Analysis, Volume II: Applications, vol. 500, Kluwer Academic, Dordrecht, The Netherlands, 2000.

[2] S. Migórski, "Existence and relaxation results for nonlinear evolution inclusions revisited," Journal of Applied Mathematics and Stochastic Analysis, vol. 8, no. 2, pp. 143-149, 1995.

[3] S. Migórski, "Existence and relaxation results for nonlinear second order evolution inclusions," Discussiones Mathematicae: Differential Inclusions, vol. 15, no. 2, pp. 129-148, 1995.

[4] N. S. Papageorgiou and N. Yannakakis, "Extremal solutions and strong relaxation for nonlinear periodic evolution inclusions," Proceedings of the Edinburgh Mathematical Society, vol. 43, no. 3, pp. 569-586, 2000.

[5] A. A. Tolstonogov, "Relaxation in nonconvex optimal control problems described by first-order evolution equations," Matematicheskiu Sbornik, vol. 190, no. 11, pp. 135-160, 1999, English translation, Sbornik Mathematics, vol. 190, pp. 1689-1714, 1999.

[6] A. A. Tolstonogov, "Relaxation in nonconvex optimal control problems with subdifferential operators," Journal of Mathematical Sciences, vol. 140, no. 6, pp. 850-872, 2007.

[7] A. A. Tolstonogov, "Relaxation in control systems of subdifferential type," Izvestiya: Matematicheskaya, vol. 70, no. 1, article 121, 2006.

[8] L. Gaul, P. Klein, and S. Kemple, "Damping description involving fractional operators," Mechanical Systems and Signal Processing, vol. 5, no. 2, pp. 81-88, 1991.

[9] W. G. Glockle and T. F. Nonnenmacher, "A fractional calculus approach to self-similar protein dynamics," Biophysical Journal, vol. 68, no. 1, pp. 46-53, 1995.

[10] R. Hilfer, Applications of Fractional Calculus in Physics, World Scientific, Singapore, 2000. 
[11] A. A. Kilbas, H. M. Srivastava, and J. J. Trujillo, Theory and Applications of Fractional Differential Equations, vol. 204 of North-Holland Mathematics Studies, Elsevier Science B.V., Amsterdam, The Netherlands, 2006.

[12] K. S. Miller and B. Ross, An Introduction to the Fractional Calculus and Fractional Differential Equations, John Wiley \& Sons, New York, NY, USA, 1993.

[13] B. Ahmad and S. K. Ntouyas, "A note on fractional differential equations with fractional separated boundary conditions," Abstract and Applied Analysis, vol. 2012, Article ID 818703, 11 pages, 2012.

[14] R. P. Agarwal, B. Ahmad, A. Alsaedi, and N. Shahzad, "On the dimension of the solution set for semilinear fractional differential inclusions," Abstract and Applied Analysis, vol. 2012, Article ID 305924, 10 pages, 2012.

[15] Y. S. Tian and A. P. Chen, "The existence of positive solution to three-point singular boundary value problem of fractional differential equation," Abstract and Applied Analysis, vol. 2009, Article ID 314656, 18 pages, 2009.

[16] Z. H. Liu and J. H. Sun, "Nonlinear boundary value problems of fractional functional integrodifferential equations," Computers \& Mathematics With Applications, vol. 64, no. 10, pp. 3228-3234, 2012.

[17] Z. Liu, J. Han, and L. Fang, "Integral boundary value problems for first order integro-differential equations with impulsive integral conditions," Computers \& Mathematics with Applications, vol. 61, no. 10, pp. 3035-3043, 2011.

[18] Y. Zhou and F. Jiao, "Existence of mild solutions for fractional neutral evolution equations," Computers E Mathematics with Applications, vol. 59, no. 3, pp. 1063-1077, 2010.

[19] Y. Zhou and F. Jiao, "Nonlocal Cauchy problem for fractional evolution equations," Nonlinear Analysis: Real World Applications, vol. 11, no. 5, pp. 4465-4475, 2010.

[20] J. Wang and Y. Zhou, "Existence and controllability results for fractional semilinear differential inclusions," Nonlinear Analysis: Real World Applications, vol. 12, no. 6, pp. 3642-3653, 2011.

[21] S. Kumar and N. Sukavanam, "Approximate controllability of fractional order semilinear systems with bounded delay," Journal of Differential Equations, vol. 252, no. 11, pp. 6163-6174, 2012.

[22] R. Sakthivel, Y. Ren, and N. I. Mahmudov, "On the approximate controllability of semilinear fractional differential systems," Computers \& Mathematics with Applications, vol. 62, no. 3, pp. 1451-1459, 2011.

[23] J. Wang and Y. Zhou, "A class of fractional evolution equations and optimal controls," Nonlinear Analysis: Real World Applications, vol. 12, no. 1, pp. 262-272, 2011.

[24] S. Hu and N. S. Papageorgiou, Handbook of Multivalued Analysis, Volume I: Theory, vol. 419, Kluwer Academic Publishers, Dordrecht, The Netherlands, 1997.

[25] J.-P. Aubin and A. Cellina, Differential Inclusions: Set-Valued Maps and Viability Theory, vol. 264, Springer, Berlin, Germany, 1984.

[26] F. Mainardi, P. Paradisi, and R. Gorenflo, "Probability distributions generated by fractional diffusion equations," in Econophysics: An Emerging Science, J. Kertesz and I. Kondor, Eds., Kluwer, Dordrecht, The Netherlands, 2000.

[27] J. Dixon and S. McKee, "Weakly singular discrete Gronwall inequalities," Zeitschrift für Angewandte Mathematik und Mechanik, vol. 66, no. 11, pp. 535-544, 1986.

[28] K. T. Andrews, "Universal Pettis integrability," Canadian Journal of Mathematics, vol. 37, no. 1, pp. 141-159, 1985.

[29] C. J. Himmelberg, “Measurable relations," Fundamenta Mathematicae, vol. 87, pp. 53-72, 1975.

[30] Q. J. Zhu, "On the solution set of differential inclusions in Banach space," Journal of Differential Equations, vol. 93, no. 2, pp. 213-237, 1991.

[31] A. A. Tolstonogov and D. A. Tolstonogov, "Lp-continuous extreme selectors of multifunctions with decomposable values: existence theorems," Set-Valued Analysis, vol. 4, no. 2, pp. 173-203, 1996.

[32] A. A. Tolstonogov and D. A. Tolstonogov, "Lp-continuous extreme selectors of multifunctions with decomposable values: relaxation theorems," Set-Valued Analysis, vol. 4, no. 3, pp. 237-269, 1996.

[33] F. Hiai and H. Umegaki, "Integrals, conditional expectations, and martingales of multivalued functions," Journal of Multivariate Analysis, vol. 7, no. 1, pp. 149-182, 1977.

[34] A. A. Tolstonogov, "On the Scorza-Dragoni theorem for multivalued mappings with a variable domain," Matematicheskie Zametki, vol. 48, no. 5, pp. 109-120, 1990, English translation, Mathematical Notes, vol. 48, pp. 1511-1158, 1990. 


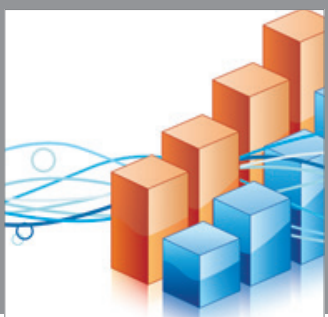

Advances in

Operations Research

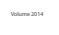

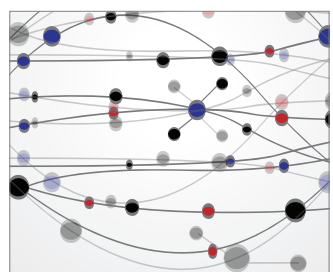

\section{The Scientific} World Journal
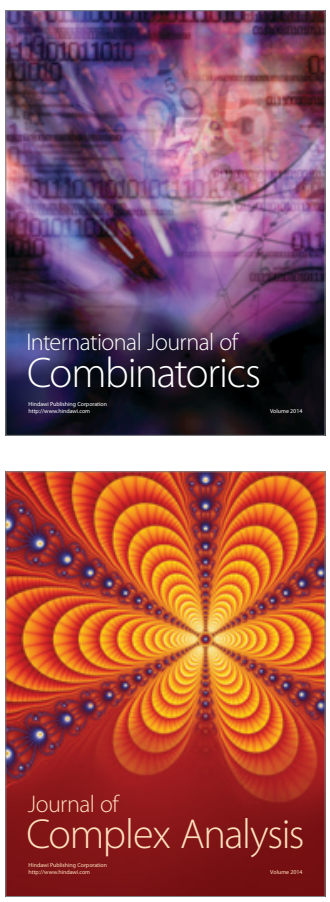

International Journal of

Mathematics and

Mathematical

Sciences
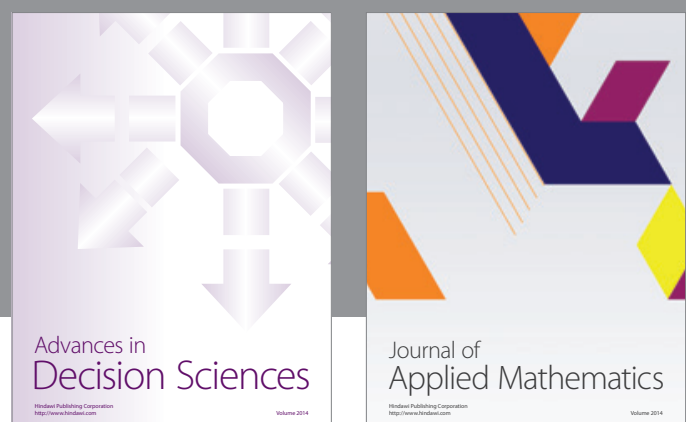

Journal of

Applied Mathematics
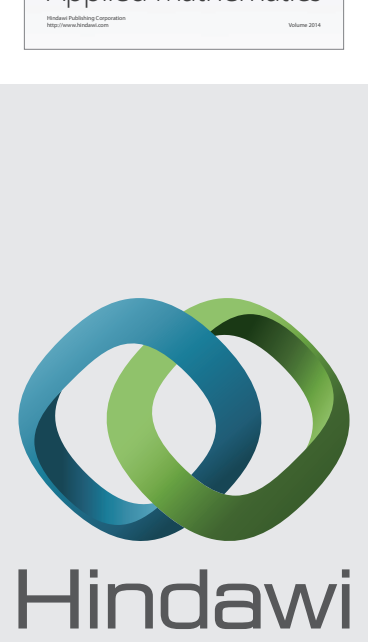

Submit your manuscripts at http://www.hindawi.com
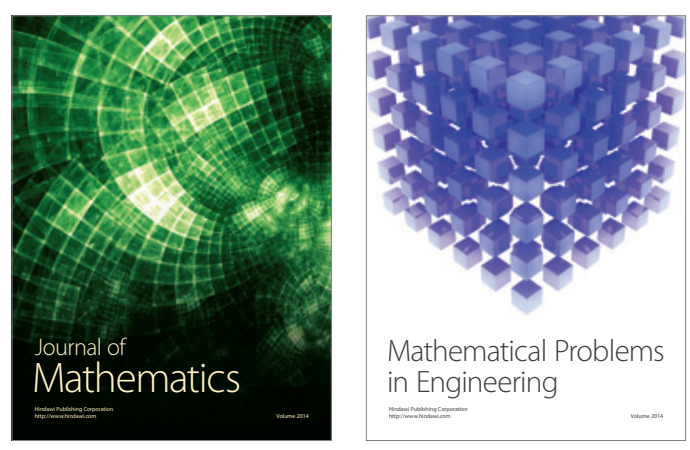

Mathematical Problems in Engineering
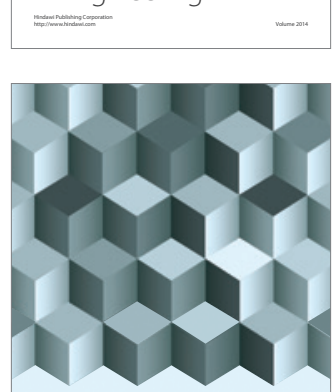

Journal of

Function Spaces
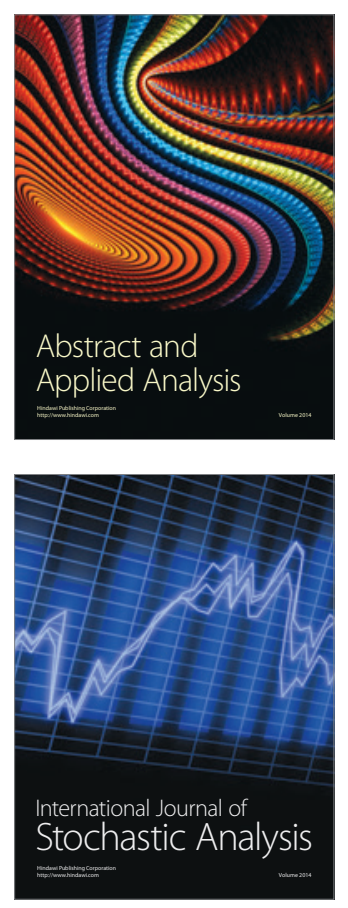

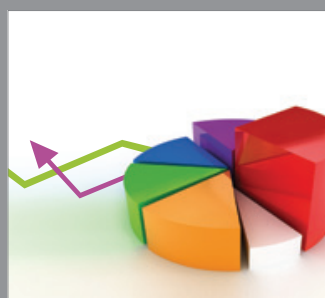

ournal of

Probability and Statistics

Promensencen
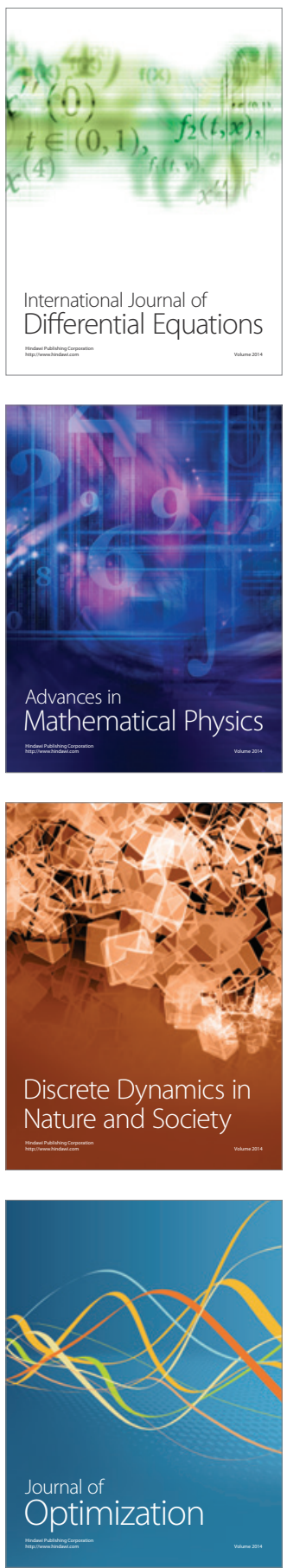